\title{
Review of "Research Counts, Not the Journal"
}

\author{
Giorgio Bedogni ${ }^{1}$ \\ 1 Fondazione Italiana Fegato ONLUS
}

Disclaimer: I am a Qeios cofounder.

Thank you for this well-researched and thought-provoking article!

I think that this article will be an excellent primer for all the researchers interested in the Journal Impact Factor (IIF). I suppose that they are many, because, as you argue in your article, the quality of the research of an increasing number of us, is being evaluated using this wrong metric.

In this article, you make it very clear that the JIF was not devised to evaluate a paper or an author, which is, very unfortunately, the way it is increasingly used. You report interesting cases of misuse of the JIF in Portugal, Czech Republic, Spain, Brazil, China, India and Ecuador, pointing to a potential "pandemic" of JIF misuse.

Both as physician and statistician, I have learnt to listen attentively to the outliers, who in your paper are expert bibliometricians and researchers awarded the Nobel Prize. I especially like your vignettes featuring Peter Doherty saying that the JIF is a "whole status thing" (Figure 3) and Brian Kobilka saying that "If you do good work, it will be published and will be cited, no matter where you publish" (Figure 7).

I have the luck of working as research methodologist and applied statistician with many researchers worldwide. Even the most talented of them are worried of publishing on journals with high JIF because their "career" depend on it. To be fair, this is more than understandable with the rewarding system currently in place for scientists.

I remember e-mails sent to me saying "I have realized my dream of publishing on X" where $X$ is a "top-journal" as determined by JIF. To which I nowadays reply by saying "Hopefully, it will be cited also!". Very few papers in a given journal attract most of its citations, as you clearly point out in your paper. Now in my replies to such e-mails, I will 
quote also Peter Doherty in Figure 3 ("the status thing").

I also remember 2 years lost submitting a good paper to clearly inappropriate journals because they had an "high JIF". How can I tell that the paper was good? Simply by the high number of citations it captured after its publication, exactly what Brian Kobilka is saying in Figure 7.

Chang ing the way scientists are evaluated and rewarded may not be simple. Listening to the outliers is, in my opinion, always a good start. So, thank you for this very nice contribution to the literature. 